\title{
Perfil del estudiante y desempeño en actividades virtuales de retroalimentación entre pares en torno a capítulos de tesis de posgrado
}

\author{
Student profile and performance in virtual peer feedback activities around postgradu- \\ ate thesis chapters
}

\author{
Hilda Difabio de Anglat \\ e-mail: hdifabio@mendoza-conicet.gob.ar \\ Consejo Nacional de Investigaciones Cientificas y Técnicas. Argentina \\ Guadalupe Álvarez (iD \\ e-mail: galvarez@ungs.edu.ar \\ Consejo Nacional de Investigaciones Cientificas y Técnicas. Argentina
}

\begin{abstract}
Resumen
Estudios centrados en las prácticas de enseñanza de la escritura en el posgrado sugieren que las propuestas basadas en el intercambio con pares y expertos (profesores y supervisores), ya sea en instancias de formación presencial como virtual, hacen posible abordar y profundizar las diferentes dimensiones (experienciales, epistemológicas y textuales) que están involucradas en la elaboración de la tesis. Con el objeto de ahondar en estos hallazgos, el presente artículo analiza las intervenciones que estudiantes con diferentes perfiles escriturales comparten en grupos de trabajo conformados por dos integrantes y un docente respecto de capítulos avanzados de las tesis de posgrado del par. La experiencia se implementa en un seminario virtual que se desarrolló durante 2017, con 90 horas de duración, en la Moodle institucional de la Facultad de Filosofía y Letras, Universidad Nacional de Cuyo. La aproximación a los perfiles se obtiene mediante varios instrumentos de diagnóstico. Se propone que los estudiantes y también el docente experto analicen los capítulos en función de tres fases, cada una de una semana de duración: la primera centrada en el modelo de la situación comunicativa y el modelo del evento; la segunda, en el modelo textual con foco en el reconocimiento de movimientos y pasos; la tercera, en el modelo textual con foco en las estrategias lingüísticas. Desde un sistema categorial exhaustivo que se aplica a un corpus de 584 comentarios, los resultados permiten reconocer similitudes y diferencias en las retroalimentaciones de los estudiantes de perfil semejante y de perfil disímil.

Palabras clave: escritura de la tesis; perfil del alumno; retroalimentación; curso postuniversitario; aprendizaje en línea.
\end{abstract}

\begin{abstract}
Studies focused on writing teaching practices at postgraduate level suggest that proposals based on the exchange with peers and experts (teachers and supervisors), whether face-to-face or through virtual training, make it possible to address and deepen the different dimensions (experiential, epistemological and textual) that are involved in the elaboration of the thesis. In order to delve into these findings, this article analyses the interventions that students with different writing profiles share in work groups formed by two members and a teacher with respect to advanced chapters of the graduate thesis of the pair. The article analyses the interventions that students with different writing profiles share in work groups formed by two members and a teacher regarding advanced chapters of the graduate thesis of the peer. The experience is implemented in a virtual seminar that was developed during 2017, with 90 hours of duration, in the institutional Moodle of the Faculty of Philosophy and Literature, National University of Cuyo. The approximation to the profiles is obtained by means of several diagnostic instruments. From a comprehensive category system that is applied to a corpus of 584 comments, the results allow to recognize similarities and differences in the feedback from students of similar profile and of dissimilar profile.

Keywords: thesis's writing; student's profile; feedback; postgraduate courses; online learning.
\end{abstract}

Recibido / Received: 18-06-2019

Aceptado / Accepted: 13-12-2019

Publicación en linea / Published online: 01-07-2020

Cómo referenciar este artículo / How to reference this article:

Difabio de Anglat, H., \& Álvarez, G. (2020). Perfil del estudiante y desempeño en actividades virtuales de retroalimentación entre pares en torno a capítulos de tesis de posgrado. Tendencias Pedagógicas, 36, pp. 26-43. doi: $10.15366 /$ tp2020.36.03 


\section{Introducción}

Dado que la investigación de posgrado se ordena al descubrimiento y producción de conocimiento inédito, se la reconoce como un área central de la innovación y el desarrollo. Por ello, las universidades y los organismos de control (e. g. la Comisión Nacional de Evaluación y Acreditación Universitaria - CONEAU - en Argentina) se esfuerzan por incrementar su calidad y eficiencia frente a la escasa eficacia terminal de los programas. Según refiere Carlino (2005), en los países de habla inglesa se llama ABD (All But Dissertation) a la situación de los estudiantes que han cumplimentado todos los requerimientos de la carrera doctoral a excepción de la tesis. En este sentido, entre los factores que entorpecen la elaboración de las evaluaciones parciales o finales de las carreras, destacan las dificultades para la escritura (Caffarella y Barnett, 2000; Castelló, González e Iñesta, 2010; Starke-Meyerring et al., 2014) y la resolución en solitario de las tareas, con ausencia o escasez de orientaciones didácticas (Delamont, 2005). Frente a este contexto, se vuelve necesario diversificar y dar mayor continuidad a las intervenciones pedagógicas orientadas a la producción de la tesis de posgrado, fundamentando estas intervenciones con investigaciones científicas.

El relevamiento bibliográfico sobre la escritura de la tesis de posgrado muestra estudios focalizados, principalmente, en diferentes dimensiones de las prácticas de enseñanza, a saber: las estrategias didácticas implementadas en seminarios o talleres de escritura de tesis a cargo de docentes expertos (Arnoux et al., 2004; Carlino, 2008; DeLyser, 2003); la dinámica de los grupos de escritura orientados específicamente a la redacción de tesis (Aitchison y Lee, 2006; Colombo, 2013; Colombo y Carlino, 2015; Lassig et al., 2009; Maher et al., 2008); el rol del supervisor, incluyendo sus formas de retroalimentación (Basturkmen, East y Bitchener, 2014; Bitchener, Basturkmen e East, 2010; Kumar y Stracke, 2007; Li y Seale, 2007; Rebollo Quintela y Espiñeira Bellón, 2017; San Mateo-Valdehíta, EscobarÁlvarez y Chacón-Beltrán, 2018). Si bien la mayoría de los estudios registrados se realizan en contextos de formación presencial, recientemente se han publicado algunas investigaciones centradas en instancias de formación virtual (Álvarez y Difabio de Anglat, 2017a, 2017b; Difabio de Anglat y Álvarez, 2017; Difabio de Anglat y Heredia, 2013; Kozar y Lum, 2015).

Entre los estudios que dan cuenta de seminarios o talleres presenciales destinados a la escritura de tesis, DeLyser (2003) ha expuesto la propia experiencia como docente en el seminario titulado «SocialScience Writing》 (Escritura en las Ciencias Sociales). Se trata de un curso avanzado para graduados en Geografía y Antropología que propone clases semanales de tres horas. Cada clase está dividida en tres secciones: primero, lectura de textos sobre escritura para ayudar a los estudiantes con el proceso y las estrategias escriturales; segundo, lectura de ejemplares en los cuales se pueda reconocer «el tema de la semana); tercero, trabajo grupal sobre las tareas de escritura asignadas con una semana de anticipación o en la misma clase. Según el autor, este seminario permite a los estudiantes mejorar sus habilidades para la escritura y transformar esta actividad en un proceso positivo y productivo para sus vidas; además, al compartir su trabajo con pares, los estudiantes desarrollan una mirada críticoconstructiva y pierden el miedo a socializar sus producciones.

A nivel nacional, Carlino (2008) describe tres situaciones didácticas implementadas, en el marco de ciclos de investigación-acción, en talleres de escritura de maestrías en Educación y Psicología: por un lado, análisis de los géneros tesis y proyecto de tesis, lo cual implica que los estudiantes deriven, a partir de diferentes ejemplares, las propiedades de los géneros; por otro lado, confección de un diario en torno a la tesis, lo que implica la escritura privada, sin convenciones; finalmente, la revisión entre pares, guiada por el docente, de los borradores de cada estudiante. Una conclusión fundamental es que los maestrandos requieren que las actividades sean andamiadas de manera externa y frecuente a fin de lograr implementarlas y reconocer su utilidad.

En cuanto a los grupos de escritura formalizados en la redacción de tesis, Maher et al. (2008) reseñan, desde los propios estudiantes, la experiencia en un grupo de escritura y ponen de manifiesto varios beneficios esenciales. En primer lugar, el tipo de aprendizaje realizado gracias a la dinámica de los alumnos aprendiendo juntos y su progresivo ingreso a miembros de una comunidad científica (Boud y Lee, 2005), dado que el conocimiento experto de los supervisores y facilitadores permite reconocer las formas de aprender y de comunicar de los demás. En segundo lugar, la práctica escritural en el marco de una comunidad discursiva específica facilita a los estudiantes la advertencia de las convenciones y protocolos propios de una audiencia académica particular según se materializan en la investigación informada en ejemplares del género en análisis. Finalmente, un aspecto central de la experiencia ha sido el tránsito desde la representación y práctica de la escritura como proceso 
implícito y esencialmente privado, a la escritura como cuestión pública y trabajo compartido; tránsito que también destacan otros autores (e. g. Carlino, 2005).

En línea con dicha dinámica de los grupos de escritura, Colombo (2013), a nivel nacional, refiere una experiencia con subgrupos de tres doctorandos en distintas disciplinas y en diferentes niveles de avance de sus tesis, que cada quince días deliberan, en una reunión presencial de dos horas de duración, sobre sus comentarios a un borrador leído de antemano o al texto ya modificado a partir de aquellos. Según la autora, esta dinámica promueve la retroalimentación mediante sugerencias específicas tanto por escrito como en el intercambio presencial; inclusive, a veces, trabajan el texto de modo conjunto para mejorarlo.

Entre los autores que se han ocupado del rol del supervisor, haciendo foco en general en sus retroalimentaciones (feedback), resulta relevante el estudio de Basturkmen et al. (2014), quienes han examinado los comentarios de retroalimentación que los supervisores realizan en los borradores de la tesis de diferentes disciplinas. En particular, se han preguntado cuál es el foco de los comentarios y cómo están formulados. En cuanto al foco, han establecido cuatro categorías: contenido, requerimientos formales, cohesión y coherencia, exactitud y adecuación lingüística. En cuanto a la función pragmática, como en Kumar y Stracke (2007), han distinguido entre comentario referencial, directivo y expresivo o apreciativo. El referencial proporciona información, corrección o incluye una reformulación. El directivo sugiere o establece futuras acciones, busca obtener información o conexiones entre ideas. El apreciativo comprende valoraciones positivas o negativas sobre el escrito.

Entre los estudios contextualizados en escenarios virtuales (Kozar y Lum, 2015; Difabio de Anglat y Heredia, 2013), Kozar y Lum (2015) discuten diferentes aspectos, incluidas las demandas pedagógicas que deben ser consideradas por las instituciones que buscan mejorar las experiencias. Así, subrayan el potencial de los grupos de escritura desarrollados mediante el uso de herramientas de comunicación mediadas por computadora.

Por su parte, Difabio de Anglat y Heredia (2013) sintetizan los principales aspectos de un taller virtual de cuatro meses dictado a través de la plataforma Moodle a fin de promover, desde un enfoque comprehensivo de la escritura científica, la producción de un capítulo escrito por el alumno como parte de sus estudios doctorales. Los resultados demuestran la mejora del proceso personal de escritura (en la reflexión, la competencia crítica, etc.) desde un enfoque didáctico que denominan «manos a la obra». También se vuelve evidente que el taller ha permitido superar uno de los problemas del estudiante de posgrado: el aislamiento académico y social.

Asimismo, en el marco de un taller virtual de escritura de tesis de posgrado basado en el trabajo entre pares, Álvarez y Difabio de Anglat (2017a) han mostrado que este tipo de trabajo permite complementar y profundizar el proceso de reflexión metalingüística en torno a la tesis (de doctorado, en esta experiencia) en tanto promueve un esfuerzo de toma de conciencia y explicitación que no sería posible en soledad. Esta profundización posibilitaría una trasposición del foco de análisis desde el nivel global y macrotextual (i. e. determinación de movimientos y pasos) al microtextual, con el establecimiento minucioso de los recursos y las estrategias lingüísticas empleados en cada sección. Además, la profundización en el análisis se vincularía con la apropiación progresiva de léxico especializado, en particular, de las Ciencias del Lenguaje. Por otra parte, señalan que la interacción grupal no se generaría de manera espontánea sino a propósito de consignas específicamente diseñadas por el docente.

En otra investigación en el contexto del taller referido supra, Álvarez y Difabio de Anglat (2018) han analizado, mediante una metodología mixta, la retroalimentación del docente en la producción de un capítulo que los estudiantes llevan a cabo como cierre del taller, así como los cambios que efectúan a partir de esa retroalimentación (feedforward). Una conclusión fundamental de este estudio es que el diagnóstico inicial de cada alumno se asocia con ese feedforward: quienes manifiestan mayor número de cambios son los que, según el perfil del diagnóstico, «planifican y revisan su texto, valoran la importancia y utilidad de la escritura, monitorean el propio desempeño, buscan selectivamente ayuda en expertos y/o en los compañeros, desarrollan sentimientos de autoeficacia como escritor» (p. 21) y, en línea con un tipo de concepción interpretativo-constructiva del conocimiento/aprendizaje, entienden la escritura en términos de elaboración. Y a la inversa: un desempeño descendido en dichas variables se corresponde con una frecuencia mayor de ausencia de cambios en los documentos.

Los trabajos citados previamente parecerían mostrar que las propuestas de enseñanza basadas en el intercambio con pares y expertos (profesores y supervisores), ya sea en instancias de formación 
presencial como virtual, hacen posible abordar y profundizar las diferentes dimensiones (experienciales, epistemológicas y textuales) que están involucradas en la elaboración de la tesis. El estudio de Álvarez y Difabio de Anglat (2017b) sugiere, además, que el intercambio con pares y expertos incidiría en la producción textual de la tesis, pero de forma diferente según los perfiles de cada estudiante. En las bases bibliográficas (e. g. EBSCO, GOOGLE Académico), sin embargo, no hemos hallado otras investigaciones que, en instancias de formación virtual en escritura de tesis de posgrado, aborden la incidencia de la dinámica de trabajo grupal con pares y expertos en los avances de cada tesista respecto del conocimiento de la tesis como género y en su producción textual concreta. Para abordar este aspecto poco estudiado, el presente artículo se propone el siguiente objetivo específico: Analizar, en el marco de actividades de intercambio y retroalimentación de pares y expertos en torno a capítulos de tesis de posgrado, las intervenciones que estudiantes con diferentes perfiles comparten en el capítulo de un par.

\section{Aspectos metodológicos}

El estudio comunicado es predominantemente cualitativo con instancias cuantitativas. En el primer sentido, se orienta a comprender fenómenos desde la perspectiva de los participantes y en relación con el contexto (Hernández Sampieri, Fernández Collado y Baptista Lucio, 2014; Stake, 2010), y asume el diseño de un estudio de casos múltiples (Stake, 2005, 2010). Siguiendo esta tradición, hemos buscado llevar adelante un análisis que capture la complejidad de los casos específicos y permita realizar comparaciones y contrastaciones que apoyen o desafíen las teorías vigentes acerca de las dinámicas de trabajo grupal y la construcción de conocimiento de los estudiantes sobre escritura de la tesis de posgrado.

Como contexto del estudio, seleccionamos casos de estudiantes que han participado de una edición de un seminario de posgrado en modalidad virtual — de 90 horas de duración —, que las mismas responsables de la investigación llevan a cabo en 2017. Este seminario — denominado «Clínica de la producción conceptual y escrita de la tesis de posgrado»— se desarrolló en la Facultad de Filosofía y Letras, Universidad Nacional de Cuyo (UNCuyo), como curso acreditable. Se destinó a profesionales de diferentes carreras de las Ciencias Sociales y Humanas: cursaron nueve doctorandos (en Educación, Letras, Psicología y Filosofía del Derecho) y un maestrando en Lingüística, siete de la UNCuyo, dos de universidades sanjuaninas y una de la Universidad del Aconcagua (Mendoza); cuatro de ellos menores de 40 años y los restantes mayores de 50; solo dos varones. Para ser aceptados en este seminario, deben contar con al menos dos capítulos de la tesis, lo cual determina que los participantes sean estudiantes avanzados de la carrera de posgrado y que ya hayan iniciado el desafío de la escritura de la tesis.

El seminario, tipo taller, comprende un entorno de formación virtual orientado a la escritura de la tesis de posgrado que combina un espacio en la plataforma Moodle de la facultad y documentos compartidos en Google Drive. Este entorno se diseña en función de una serie de supuestos didácticos, pedagógicos y tecnológicos, que se detallan en publicaciones previas (Álvarez y Difabio de Anglat, 2016, 2017a, 2017b, 2018; Difabio de Anglat y Álvarez, 2017).

Es importante destacar que el taller se organiza en función de tres etapas.

Durante la etapa inicial, en dos foros diferentes creados en el espacio virtual que el taller abrió en la plataforma Moodle institucional, se solicita a los estudiantes que, por un lado, se presenten y, por otro, reflexionen sobre el proceso de escritura, especialmente la escritura de la tesis, y sobre la importancia de aceptar sugerencias constructivas y de comprometerse a proporcionarlas al compañero/a.

En la segunda etapa, son divididos por afinidad disciplinar o vinculación genérica de los temas que investigan en 5 (cinco) grupos de dos integrantes, en el marco de los cuales se les propone que analicen los capítulos producidos por cada integrante teniendo en cuenta, fundamentalmente, el Modelo de situación comunicativa, el Modelo de evento y el Modelo textual subyacente (Cubo de Severino y Bosio, 2011, pp. 29-30). El primero refiere al rol de los participantes en la interacción y su contexto, a los propósitos, expectativas y conocimientos del autor y de la audiencia potencial. El modelo de evento, al contenido sobre el que trata el texto, al encuadre - teórico y metodológico- distintivo de la investigación, a la creación de nuevo conocimiento. El modelo textual remite a las estrategias de formulación escrita de los significados que se busca comunicar, «tanto a nivel global en los diferentes 
movimientos discursivos (superestructura y macroestructura de la tesis) como a nivel local (microestructura de la tesis)» (p. 30).

Así, se organiza este análisis en tres fases, cada una de una semana de duración. La primera, centrada en el modelo de la situación comunicativa y en el modelo del evento; la segunda fase, en el modelo textual, particularmente en el reconocimiento de movimientos y pasos de los capítulos específicos $^{1}$; y la tercera fase, en los recursos y las estrategias lingüísticas propios también de cada capítulo de la tesis.

Para llevar a cabo el análisis, se genera, por cada uno de los capítulos, un documento compartido en Google Drive, en el cual cada estudiante comparte su capítulo, y un foro, en el que inicialmente cada uno sintetiza las características básicas de la investigación que lleva a cabo. A su vez, en cada fase se comparte en la plataforma Moodle un documento elaborado ad hoc sobre la temática por trabajar y bibliografía vinculada. El análisis involucra al menos dos tipos de consignas fundamentales: por un lado, aquellas que apuntan a leer el capítulo e ir señalando logros o inadecuaciones de cada fragmento y sección en relación con cada modelo; por otro, las que están orientadas a elaborar una reflexión más general sobre los logros o inadecuaciones del capítulo. Así, las tareas requeridas apuntan a desarrollar la retroalimentación (R) en texto y la R global (Kumar y Stracke, 2007) sobre el capítulo del colega del equipo y sobre el propio. La $\mathrm{R}$ en texto refiere a todos los comentarios escritos en el texto, en este caso en los márgenes del documento compartido en Google Drive, que se pueden describir mejor como pensamientos espontáneos, que expresan el diálogo que va estableciendo quien lee con el autor. La R global adquiere la forma de un mensaje, en el cual el par o el supervisor sintetizan sus principales apreciaciones respecto de todo el texto, así como de los apartados individuales. La R globales se comparten en el foro de cada capítulo.

En la última etapa del curso, se destinan dos semanas para que cada estudiante revise el propio capítulo en función de los comentarios generados por el par. Durante este lapso, se implementan vía correo electrónico instancias de intercambio y retroalimentación con las docentes y los directores de tesis.

A los fines del diagnóstico, utilizamos: 1) un cuestionario, de elaboración propia, denominado «En torno a la escritura de la tesis» (cuyas preguntas remiten a: particularidades del proceso de escritura de la tesis doctoral; grado de avance; estrategias implementadas para la planificación, la puesta en texto y la revisión; dispositivos, programas y aplicaciones empleados; dificultades que ha enfrentado y desafío actual; rol de otros investigadores y/o compañeros del posgrado en el proceso personal de tesis; el contenido de este instrumento fue validado por un grupo de tres especialistas), 2) un inventario de escritura académica en el posgrado (Difabio de Anglat, 2012), 3) un cuestionario sobre concepciones de aprendizaje/conocimiento, en el cual combinamos el CONAPRE — cuestionario sobre Concepciones de Aprendizaje (Martínez-Fernández, 2004, 2007); una escala Likert de 15 ítems - con seis ítems de la subescala Modelos mentales del Inventory Learning Style -ILS por su sigla en inglés(Vermunt, 1994) y 4) un cuestionario que pondera, entre otros aspectos, concepciones de investigación (Difabio de Anglat et al., 2018).

Como evaluación final de la intervención, utilizamos dos instrumentos: 1) cuestionario retrospectivo de preguntas abiertas, elaborado ad hoc, que busca ponderar la valoración de los estudiantes en torno a los aportes y limitaciones del taller y su efecto en la conformación del habitus científico (Bourdieu, 1976), de la identidad como investigadores y escritores ${ }^{2}$ y 2) segunda aplicación del inventario de escritura académica en el posgrado.

\footnotetext{
${ }^{1}$ El término movimiento — move — «captura el propósito comunicativo de un segmento textual en un nivel más general, el paso — step — explica más expresamente el medio retórico de la realización de la movida. Una movida puede realizarse mediante un solo paso retórico o por la combinación de varios» (Jara Solar, 2013, p. 77).

${ }^{2}$ El cuestionario incluye preguntas orientadas a revelar información sobre las experiencias de posgrado previas a la Clínica que han favorecido la producción textual, el alcance de la Clínica y su dinámica (destacando la retroalimentación entre pares) en esa producción y en las autopercepciones del estudiante como escritor académico, así como el rol de la mediación
} 
En este artículo nos centramos en un aspecto poco trabajado aún en la bibliografía: el perfil del estudiante y su desempeño en actividades de intercambio y retroalimentación de pares y expertos en torno a capítulos de tesis; específicamente, analizamos la retroalimentación de los participantes al capítulo del par. A continuación, detallamos de qué manera se ha llevado a cabo la caracterización del perfil de los cursantes y el análisis de sus intervenciones en los espacios de intercambio seleccionados.

A fin de conformar una aproximación al perfil del estudiante derivado del diagnóstico, en primer lugar, establecimos - mediante el cálculo de la correlación bivariada (rho de Spearman) en el grupo total (los 74 alumnos que han participado de nuestros talleres) - las asociaciones entre las variables continuas, esto es, entre las dimensiones del inventario de escritura académica y las concepciones de aprendizaje/conocimiento; consignamos aquellas que mostraron asociación significativa (cfr. Tabla 1):

Tabla 1.

Coeficientes de correlación bivariada

\begin{tabular}{|c|c|c|c|c|c|c|c|c|c|c|c|c|c|}
\hline & 2 & 3 & 4 & 5 & 6 & 7 & 8 & 9 & 10 & 11 & 12 & 13 & 14 \\
\hline $\begin{array}{l}\text { 1. Puesta en } \\
\text { texto }\end{array}$ &, $24^{*}$ &, $51 * *$ &, $56 * *$ & ------ &, $46^{* *}$ &, $54 * *$ & ----- & ,29* &, $50 * *$ &, $47 * *$ & ------ &, $54 * *$ &, $54 * *$ \\
\hline 2. Revisión & &, $31 * *$ &, $37 * *$ &, $36 * *$ &, $25^{*}$ &, $37 * *$ & ----- & ------ & ------ & ------ & ------ & ,38* &, $41 * *$ \\
\hline 3. Valor tarea & & &, $49 * *$ &, $32 * *$ &, $43^{* *}$ &, $56 * *$ & ----- & ------ &, $43^{* *}$ &, $35 * *$ & ------ &, $47 * *$ &, $66^{* *}$ \\
\hline 4. Control & & & & ------ &, $56 * *$ &, $48^{* *}$ & ----- &, $44 * *$ &, $52 * *$ &, $40 * *$ &, $33 * *$ &, $31 * *$ &, $40 * *$ \\
\hline 5. Asistencia & & & & & ------ &, $25^{*}$ & ----- & ------ & ------ & ------ & ------- & ------ & ------ \\
\hline $\begin{array}{l}\text { 6. Ambiente- } \\
\text { tiempo }\end{array}$ & & & & & &, $45^{* *}$ & ----- &, $37 * *$ &, $38^{* *}$ &, $38 * *$ &, $27^{*}$ & ------- &, $34 * *$ \\
\hline $\begin{array}{l}\text { 7. Esc. como } \\
\text { elaboración }\end{array}$ & & & & & & & $\begin{array}{l}----- \\
-\end{array}$ &, $36 * *$ &, $52 * *$ &, $37 * *$ & ------ &, $43^{* *}$ &, $53 * *$ \\
\hline $\begin{array}{l}\text { 8. Esc. como } \\
\text { reproducción }\end{array}$ & & & & & & & & ------- & ----- & ------ &, $41 * *$ & ------ & ------ \\
\hline $\begin{array}{l}\text { 9. Autoper- } \\
\text { cepción posi- } \\
\text { tiva }\end{array}$ & & & & & & & & &, $56 * *$ &, $29 *$ & ------ &, $34 * *$ &, $29 *$ \\
\hline 10. Logros & & & & & & & & & &, $57 * *$ &, $30 * *$ &, $52^{* *}$ &, $47 * *$ \\
\hline 11. Retórica & & & & & & & & & & & ------- &, $33 * *$ &, $45^{* *}$ \\
\hline 12. Directa & & & & & & & & & & & & ------- & ------ \\
\hline $\begin{array}{l}\text { 13. Interpreta- } \\
\text { tiva }\end{array}$ & & & & & & & & & & & & &, $69 * *$ \\
\hline $\begin{array}{l}\text { 14. Construc- } \\
\text { tiva }\end{array}$ & & & & & & & & & & & & & 1 \\
\hline
\end{tabular}

Hemos destacado en color azul los índices que significan, al menos, una correlación positiva media (alrededor de 0,50; cfr. Hernández Sampieri et al., 2014, p. 305) y en amarillo el casillero de la única asociación que alcanza Escritura como reproducción.

tecnológica en los procesos de enseñanza y aprendizaje propuestos en este taller. 
Luego seleccionamos aquellas dimensiones de las estrategias que evalúa el inventario de escritura académica que evidencian más correlaciones medias con otras (Puesta en texto y Logros $3: 8$ y 7, respectivamente) y, entre las restantes de menor número (entre 3 y 5), la dimensión que mejor discrimina a los estudiantes que participan de este análisis (Retórica) ${ }^{4}$. Como resultado de este procedimiento ingresan a la aproximación al perfil una estrategia de escritura (Puesta en texto) y dos de autoeficacia escrituraria (Logros y Retórica).

A ello se suman las Concepciones de escritura — como elaboración, proceso de composición que corresponde a la producción experta, y como reproducción, modelo inmaduro (Scardamalia y Bereiter, 1992) - y las Concepciones de conocimiento/aprendizaje: directa, interpretativa y constructiva (Pozo y Scheuer, 1999), esto es, una concepción reproductiva (el conocimiento es copia fiel de la realidad y el aprendizaje consiste en recibir y recordar), una que reconoce la actividad mental como instancia mediadora pero sin alejarse del modo de presentación del contenido o la que concibe los procesos de aprendizaje formalizados en una función transformadora del conocimiento y del aprendiz.

Se categorizan además dos respuestas a preguntas abiertas de los otros instrumentos (cuestionario sobre concepciones de investigación y del denominado «En torno a la escritura de la tesis»):

a) Definición personal de investigación científica, en tres categorías en función del esclarecimiento sobre qué «cuenta» como conocimiento, reflexión, argumentación en una comunidad socio-retórica y qué métodos lo generan y garantizan: no responde o reproductiva (proporciona una noción general) ${ }^{5}$, interpretativa (manifiesta una formulación explicativa) — con tres subcategorías: básica, moderadamente elaborada y elaborada $-{ }^{6}$ y constructiva (evidencia una conceptualización transformadora) 7 .

\footnotetext{
${ }^{3}$ Logros refiere a la confianza en habilidades específicas de escritura, esto es, expectativas de competencia que se basan en experiencias de dominio real (capacidad para elaborar buenas oraciones o buenas síntesis o buenas conclusiones personales). ${ }^{4}$ Retórica también remite a la autoconfianza en habilidades específicas de escritura, pero en este caso vinculadas directamente con la capacidad de estar consciente del lector (e. g. empleo de recursos retóricos que promuevan el interés).

5 «Es una experiencia personal, que transforma las maneras de estudiar y de seguir aprendiendo.»

${ }^{6}$ Interpretación básica: «La investigación en las ciencias sociales implica un estudio minucioso sobre una problemática que no ha sido abordada; por lo tanto, la especificidad del tema es importante, junto con el conocimiento de los antecedentes.» Moderadamente elaborada: «Es el estudio sistemático de un fenómeno, de modo de poder describirlo y explicarlo de manera científica, con el objetivo de contribuir a la ciencia, al área de estudio que lo engloba. Más allá de una tarea personal, lo veo como un aporte a la comunidad científica, que se nutre de las investigaciones particulares de sus integrantes.» Elaborada: «La considero un conjunto sistemático de procedimientos que analizan un área susceptible de ser explicada, ya por primera vez, ya de una manera nueva, que represente una mejora en su conceptualización, destinada a formar parte de un grupo de conocimientos colectivos acumulables. El área de análisis necesita la conformación de un corpus (un "recorte") cuyas características permitan la intervención del analista; y los resultados del análisis deben ser expuestos de un modo lógico, sistemático y preciso.»

7 «Lo que voy observando a medida que desarrollo mi tesis es que, mientras el horizonte especulativo se va ampliando, voy "asiendo" algunos mecanismos necesarios para cumplir con lo que se me pide: deslindar los principales caminos recorridos y hacer el propio. Este camino personal debe derivar en un producto que muestre un conocimiento profundo del campo disciplinar trabajado, a la vez que señale qué metodología considero la más apropiada (recorrida o no) de la investigación
} 
b) Particularidades de la escritura de la tesis doctoral, categorizada con los mismos códigos explicados supra.

Las agrupaciones resultantes son (cfr. Tabla 2):

Tabla 2.

Criterios de definición de las tres categorias de perfil de estudiantes

\begin{tabular}{lccc}
\hline & Categoría inferior & Categoría intermedia & Categoría superior \\
\hline Puesta en texto & Baja & Media & Alta \\
Logros & Bajos & Medios & Altos \\
Retórica & Baja & Media & Alta \\
Escritura como elaboración & Baja & Media & Media o alta \\
Escritura como reproducción & Media o alta & Baja o media & Baja \\
Concepción directa & Media o alta & Baja o media & Baja o media \\
Concepción interpretativa & Baja & Media o alta & Alta \\
Concepción constructiva & Baja & Media o alta & Media o alta \\
$\begin{array}{l}\text { Definición personal de investiga- } \\
\text { ción científica }\end{array}$ & No responde o & Interpretativa & Constructiva \\
$\begin{array}{l}\text { Particularidades de la escritura de } \\
\text { la tesis doctoral }\end{array}$ & reproductiva & & \\
\hline
\end{tabular}

Fuente: elaboración propia.

En función de los criterios establecidos, seleccionamos cinco estudiantes de diferentes perfiles cuyas producciones se analizan. La aplicación de los criterios arriba descritos a los resultados del diagnóstico de dichos cursantes lleva a ubicarlos en el siguiente orden:

1) Estudiante 1: evidencia un perfil combinado de categorías inferiores e intermedias, en cuanto se ubica en el grupo inferior en Puesta en texto y Escritura como elaboración, informa puntuaciones medias en Logros, Retórica y Escritura como reproducción, su patrón de Concepciones de conocimiento/aprendizaje es, en cierta medida, indefinido o ambivalente ya que en las tres categorías evidencia puntajes medios, no responde la pregunta sobre Definición personal de investigación científica y es interpretativa elaborada su formulación de las Particularidades de la escritura de la tesis. En consecuencia, lo adscribimos al Perfil Inferior-Intermedio.

2) Estudiante 2, un perfil intermedio: puntuaciones medias en Puesta en texto, Logros y Escritura como elaboración, alta en Retórica, pero media en Escritura como reproducción y alto el valor en Concepción directa, al tiempo que son bajas las puntuaciones que informa en las otras dos categorías de Concepciones de conocimiento/aprendizaje. Respecto de las dos respuestas abiertas, alcanza el nivel interpretativo.

3) Estudiante 3, un perfil combinado de categorías intermedias y superiores al que se suma una categoría inferior: puntuación alta en Puesta en texto, medias en Logros y Retórica; respecto de las concepciones, evidencia ponderación alta en Escritura como elaboración y como reproducción, baja en concepción directa, media en interpretativa y alta en constructiva. Son interpretativas moderadamente elaboradas tanto su definición de investigación científica (en cuanto, si bien desarrolla una conceptualización relevante, la circunscribe al enfoque de su investigación doctoral) como su exposición de las particularidades de la tesis. Esto es, desde la «epistemología personal» (Hofer, 2004), podría ubicarse en el nivel interpretativo-constructivo; no obstante, dado el puntaje alto en Escritura como reproducción se adscribe al perfil intermedio.

lingüística. Por otra parte, es una suerte de primera "prueba de fuego" para validarme como investigadora seria en la comunidad científica.» 
4) Estudiante 4, un perfil combinado de categorías intermedias y superiores (Intermedio-Superior): puntuaciones altas en Puesta en texto, Logros y Retórica, media en Escritura como elaboración y baja en Escritura como reproducción; por otra parte, su epistemología personal se define en el nivel interpretativo (alto puntaje solo en la concepción correspondiente y formulaciones interpretativas moderadamente elaboradas en ambas respuestas a los interrogantes abiertos).

5) Estudiante 5, resulta clara su ubicación en la categoría superior: puntuaciones altas en las tres estrategias y en Escritura como elaboración —al tiempo que informa un puntaje bajo en Escritura como reproducción-; su epistemología personal se define en el nivel constructivo (alto puntaje solo en esta categoría de Concepciones de conocimiento/aprendizaje y proposiciones constructivas como respuesta a las preguntas abiertas).

En cuanto a las intervenciones analizadas, corresponden a las diferentes retroalimentaciones que cada participante ha incluido sobre el capítulo ajeno en el documento compartido correspondiente, así como en los foros. Analizamos tanto las retroalimentaciones en el texto como las globales. El análisis cualitativo de estas intervenciones ha involucrado tres fases.

En primer lugar, especificamos si la intervención está encadenada (o no) a los aportes previos del par o de la docente, es decir, si dialoga con ellos, lo cual en este corpus no ha resultado significativo debido al número mínimo de intervenciones encadenadas. Luego, consideramos la naturaleza o foco de los comentarios —orientados al modelo de situación comunicativa, al modelo de evento o al modelo textual (Cubo de Severino y Bosio, 2011) — y su función — según la categorización propuesta por Basturkmen et al. (2014): comentario referencial, directivo y apreciativo-. Finalmente, distinguimos tipos de análisis teniendo en cuenta dos criterios: por un lado, si el análisis involucra un fragmento puntual del escrito y los elementos implicados en ese fragmento (por ejemplo, una coma en una oración) o una dimensión general del escrito (por ejemplo, los signos de puntuación o el uso de las comas); por otro lado, si el análisis está (o no) fundamentado, independientemente de si la fundamentación se realiza (o no) con bibliografía sugerida en el curso. De esta manera, consideramos el siguiente sistema categorial (cfr. Tabla 3):

Tabla 3.

Categorías de análisis de los comentarios

$$
\text { Ejemplos tomados del corpus }
$$

\begin{tabular}{|c|c|c|}
\hline \multirow[t]{3}{*}{ Foco } & $\begin{array}{l}\text { Modelo de situación co- } \\
\text { municativa }\end{array}$ & $\begin{array}{l}\text { «Puede ser conveniente reformular los títulos de los apartados, ya que es } \\
\text { importante demostrar al jurado y al lector especializado que uno se aleja } \\
\text { de una lectura un tanto repetitiva del autor para abordar la comprensión } \\
\text { hermenéutica y profundizar en su análisis.» }\end{array}$ \\
\hline & Modelo de evento & $\begin{array}{l}\text { «Recomiendo aquí explicitar en qué obras el autor expone su pensa- } \\
\text { miento educativo, de modo que luego se proceda a su análisis.» }\end{array}$ \\
\hline & Modelo textual & $\begin{array}{l}\text { «Se hace una presentación del tema en general; no veo explicitado el or- } \\
\text { den de los núcleos temáticos que planteará el capítulo.» }\end{array}$ \\
\hline \multirow[t]{3}{*}{ Función } & Referencial & «Oración muy extensa.» \\
\hline & Directivo & $\begin{array}{l}\text { «Este párrafo marca el inicio de la descripción/interpretación de los tex- } \\
\text { tos. Me pregunto si no debería introducirse de un modo particular, dado } \\
\text { que inicia una secuencia de distintos análisis que construyen la visión } \\
\text { planteada más arriba.» }\end{array}$ \\
\hline & Apreciativo & $\begin{array}{l}\text { «En cuanto al modelo de situación comunicativa, el capítulo se adecua al } \\
\text { nivel de complejidad discursiva requerida para una tesis doctoral en el } \\
\text { área de Lingüística. Se advierte un uso desenvuelto de términos propios } \\
\text { de su especialización y la claridad en la exposición de los fenómenos por } \\
\text { ser estudiados. También se observa una síntesis lograda de la bibliografía } \\
\text { relevante.» }\end{array}$ \\
\hline \multirow[t]{2}{*}{$\begin{array}{l}\text { Retroalimenta- } \\
\text { ción }\end{array}$} & $\begin{array}{l}\text { Básica de un fragmento } \\
\text { puntual }\end{array}$ & $\begin{array}{l}\text { «Quizá en una nota al pie de página deberías explicar más extensamente } \\
\text { cuáles son principios o concepciones de la pedagogía de la interculturali- } \\
\text { dad.» }\end{array}$ \\
\hline & $\begin{array}{l}\text { Fundamentada de un frag- } \\
\text { mento puntual }\end{array}$ & $\begin{array}{l}\text { «Quizá puede ponerse un punto seguido porque se sigue hablando del } \\
\text { tema de diversidad y heterogeneidad.» }\end{array}$ \\
\hline
\end{tabular}


Básica de una dimensión general

Fundamentada de una dimensión general
«Me pareció interesante y clara la descripción histórica y la transformación del paisaje. Entiendo que las dos primeras páginas son introductorias, pero considero que son demasiado abarcativas de lo nacional.»

«Modelo del evento: Por lo que entiendo, este capítulo se encarga de la revisión del estado de la cuestión de la formación de los indefinidos compuestos y no introduce todavía el propio análisis del corpus. Este rasgo del capítulo puede hacerle caer en el riesgo de parecer una mera reseña de otros autores. Si bien el capítulo acierta en una presentación lógica, clara y sintética de los aportes de distintos lingüistas acerca del tema de la formación de los indefinidos compuestos, no termina de quedar clara para el lector la posición del autor de la tesis respecto de las distintas teorías de formación, o si todo el conjunto conforma una serie argumental sobre la que se sustentaría la posición del tesista (es probable, sin embargo, que esto ya esté claro en el Planteo del problema e Hipótesis del capítulo II, lo cual sería conveniente retomar). Sobre todo en la última sección, al presentar los parámetros, no queda claro si todo ello es teoría de Company o algunos de ellos son fruto del análisis del propio autor. De ser solo la teoría de Company, esta sección da al lector el efecto de ser una transcripción de la teoría de otro, en la que no queda clara la pertinencia para el propio trabajo o un grado de elaboración de estos temas sobre el propio corpus que aporte a dicha teoría o bien la cuestione.»

Fuente: elaboración propia.

Es importante destacar que, al interior de las globales, se reconocen una o más retroalimentaciones dependiendo de si el comentario alude a uno o más modelos. Por ejemplo, si remite solo al modelo textual se reconoce una retroalimentación, mientras que si, además, se refiere al modelo del evento, se reconocen dos retroalimentaciones.

Con el objeto de respaldar la consistencia de los resultados, la segunda autora de este artículo efectuó el análisis completo del corpus desde las categorías predefinidas; luego, la primera autora a la manera de una validación inter-juez - revisó dicho análisis; por último, deliberaron sobre los casos en que diferían hasta alcanzar un acuerdo firme.

Una vez cumplido este proceso y verificado el análisis de las retroalimentaciones que cada participante ha incluido sobre el capítulo ajeno en el documento compartido correspondiente y en los foros, cuantificamos estas retroalimentaciones según el foco, la función y el tipo de análisis, y comparamos los resultados de los diferentes estudiantes y sus perfiles.

El corpus se integró de 555 comentarios de $\mathrm{R}$ en texto, con una gran dispersión (rango entre 28 y 331; D.S. = 95,7); y 29 de R global; esto es, un total de 584 comentarios.

Los datos obtenidos se analizan descriptivamente mediante tablas y gráficos. Luego, por un lado, se aplica la prueba de diferencia de proporciones por medio del programa STATS (Hernández Sampieri et al., 2014), para ponderar si aparecen diferencias estadísticamente significativas entre las categorías en análisis y, por el otro, se indaga si se verifica asociación entre las variables aproximación al perfil del estudiante y $\mathrm{R}$ básica vs. fundamentada mediante $\mathrm{Chi}^{2}$ en SPSS 22.0. De esta manera, pudimos establecer similitudes y diferencias entre las retroalimentaciones de los estudiantes de perfil similar y de perfil disímil.

\section{Resultados}

Organizamos la presentación de los resultados en un esquema tripartito: en primer lugar, las retroalimentaciones en texto; luego, las globales; finalmente, la asociación entre la aproximación al perfil de cada cursante y los tipos de comentarios.

Es importante destacar que en el caso de la $\mathrm{R}$ en texto desestimamos la categoría «encadenada o no» porque, salvo un comentario, todos corresponden a «no encadenados».

\subsection{Retroalimentación en texto}

Respecto de todas las retroalimentaciones en texto se halla (cfr. Tabla 4 y Figura 1): 
Tabla 4.

Resultados descriptivos por categoría de análisis en retroalimentación en texto

\begin{tabular}{|c|c|c|c|c|}
\hline \multicolumn{3}{|c|}{ Categorías } & Frecuencia & Porcentaje \\
\hline \multirow[t]{4}{*}{ Modelo } & Textual & & 466 & $84 \%$ \\
\hline & De evento & & 75 & $13,5 \%$ \\
\hline & De situación comunicativa & & 14 & $2,5 \%$ \\
\hline & & Total & 555 & $100 \%$ \\
\hline \multirow{4}{*}{$\begin{array}{l}\text { Función prag- } \\
\text { mática }\end{array}$} & Referencial & & 37 & $6,7 \%$ \\
\hline & Directiva & & 505 & $91 \%$ \\
\hline & Apreciativa & & 13 & $2,3 \%$ \\
\hline & & Total & 555 & $100 \%$ \\
\hline \multirow[t]{3}{*}{ Tipo de análisis } & Retroalimentación básica & & 420 & $75,7 \%$ \\
\hline & Retroalimentación fundamentada & & 135 & $24,3 \%$ \\
\hline & & Total & 555 & $100 \%$ \\
\hline
\end{tabular}

Fuente: elaboración propia a partir de SPSS 22.0.

Figura 1.

Porcentajes por categorías de análisis en retroalimentación en texto

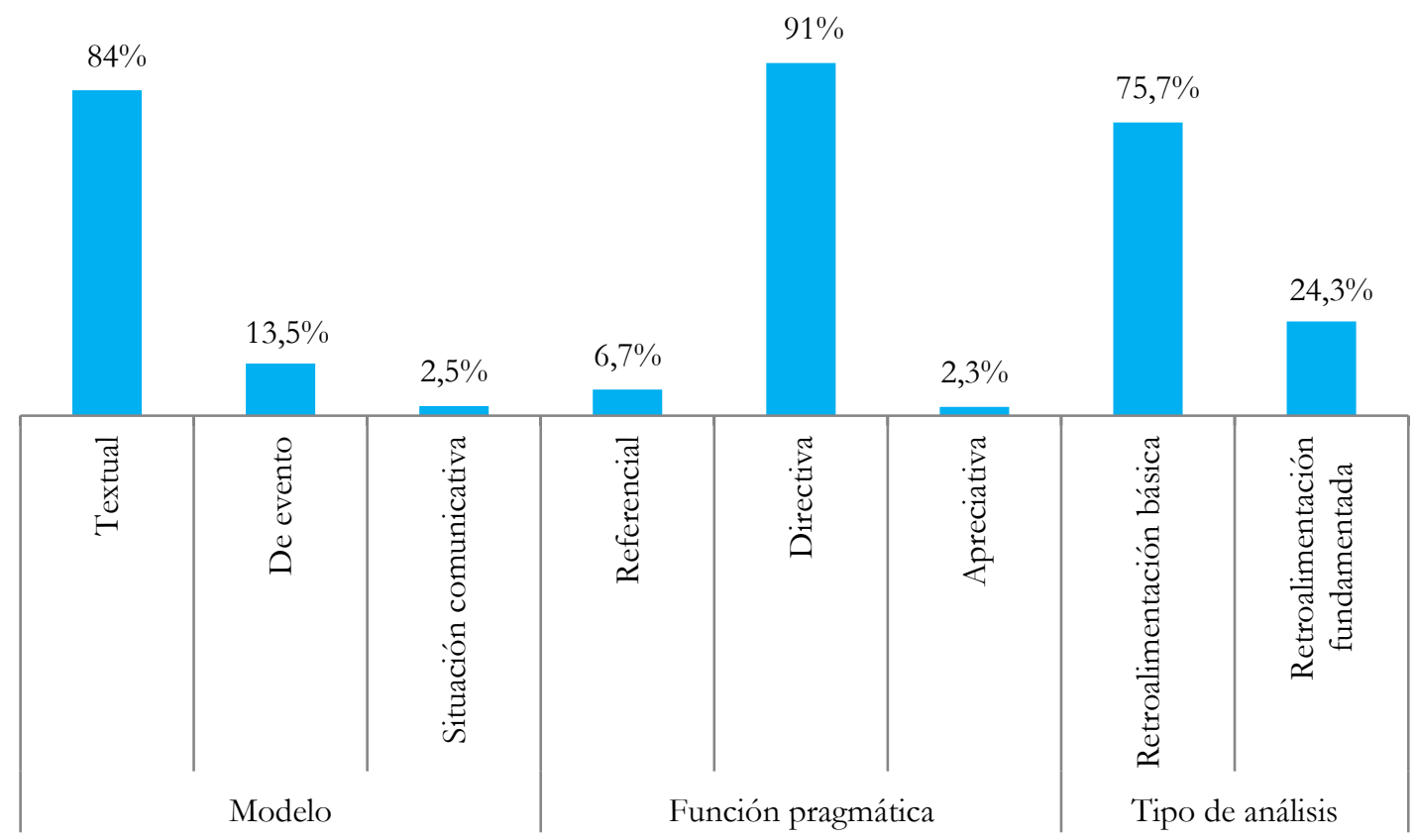

Fuente: elaboración propia.

Es evidente que priman los comentarios referidos al modelo textual —a las estrategias de formulación escrita de los significados que se busca comunicar-, con función directiva (e. g. «Convendría reforzarlo con algún ejemplo», «Cuidar la repetición de términos...») y desde una R básica (e. g. «Pienso que tendrías que configurar todas las citas según APA»); esto es, en el diálogo que establecen con el capítulo del par sugieren modificaciones puntuales.

Por otra parte, el $96 \%$ de los comentarios atiende a aspectos lingüísticos y el $4 \%$ restante a movimientos y pasos retóricos. Entre los primeros, se señalan problemas de concordancia, de régimen proposicional (e. g. «Es de acuerdo con»), de vocabulario (e. g. «¿Se puede usar un término más "técnico" o específico?») y repetición lexical (e. g. «Este vocablo también comienza a aparecer de un 
modo algo repetitivo; revisaría»), marcadores del discurso y estilo (e. g. «Se observa un estilo segmentado de períodos breves y párrafos cortos. Puede ser necesario elaborar una sintaxis más articulada a través del uso de conectores, anticipación de la información, etc.»), entre otros.

Los pocos comentarios relativos a movimientos y pasos retóricos concretan la función referencial (e. g. «Introducción clara pero algo extensa; recién aquí aborda el aspecto por desarrollar») y, con menor frecuencia, la directiva (e. g. «Aquí se presenta el orden de los núcleos temáticos que tratará el capítulo. Sugiero adaptar su explicitación a la secuencia de los títulos que se exponen a lo largo del capítulo»).

La R correspondiente al modelo de evento, si bien menos importante en número, resulta cualitativamente significativa porque se orienta a enfatizar, mediante un análisis fundamentado, aspectos que - en caso de ser atendidos - afectan la macroestructura de un apartado o, inclusive, de todo el capítulo; por ejemplo: «Conviene abordar primero fuertemente la conceptualización de "despliegue integral", para luego exponer la idea de "despliegue parcial". Es importante porque la formación integral es el meollo de la tesis. En este sentido, cabe darle al tema una hermenéutica más exhaustiva (...)».

Como es de esperar, estas diferencias alcanzan significatividad estadística ya desde la comparación con la segunda frecuencia de cada categoría (a un nivel de significación de $\mathrm{p}=0,01$, los coeficientes $\mathrm{z}$ hallados son, respectivamente: 16,$787 ; 20,165 ; \mathrm{y} 12,363)$.

\subsection{Retroalimentación global}

En relación con las retroalimentaciones globales, los resultados son (cfr. Tabla 5 y Figura 2):

Tabla 5.

Resultados descriptivos por categoría de análisis en retroalimentación global

\begin{tabular}{|c|c|c|c|c|}
\hline \multicolumn{3}{|c|}{ Categorías } & Frecuencia & Porcentaje \\
\hline \multirow[t]{3}{*}{ Interacción } & Encadenada & & 2 & $7 \%$ \\
\hline & No encadenada & & 27 & $93 \%$ \\
\hline & & Total & 29 & $100 \%$ \\
\hline \multirow[t]{4}{*}{ Modelo } & Textual & & 15 & $47 \%$ \\
\hline & De evento & & 11 & $34 \%$ \\
\hline & De situación comunicativa & & 6 & $19 \%$ \\
\hline & & Total & $32^{8}$ & $100 \%$ \\
\hline \multirow{4}{*}{$\begin{array}{l}\text { Función prag- } \\
\text { mática }\end{array}$} & Referencial & & ------ & ---- \\
\hline & Directiva & & 23 & $72 \%$ \\
\hline & Apreciativa & & 9 & $28 \%$ \\
\hline & & Total & 32 & $100 \%$ \\
\hline \multirow[t]{3}{*}{ Tipo de análisis } & Retroalimentación básica & & 9 & $31 \%$ \\
\hline & Retroalimentación fundamentada & & 20 & $69 \%$ \\
\hline & & Total & 29 & $100 \%$ \\
\hline
\end{tabular}

Fuente: elaboración propia a partir de SPSS 22.0.

\footnotetext{
${ }^{8}$ En esta categoría y en la siguiente el total se incrementa porque tres comentarios se adscribieron a dos modelos o a dos funciones pragmáticas.
} 
Figura 2.

Porcentajes por categorias de análisis en retroalimentación global

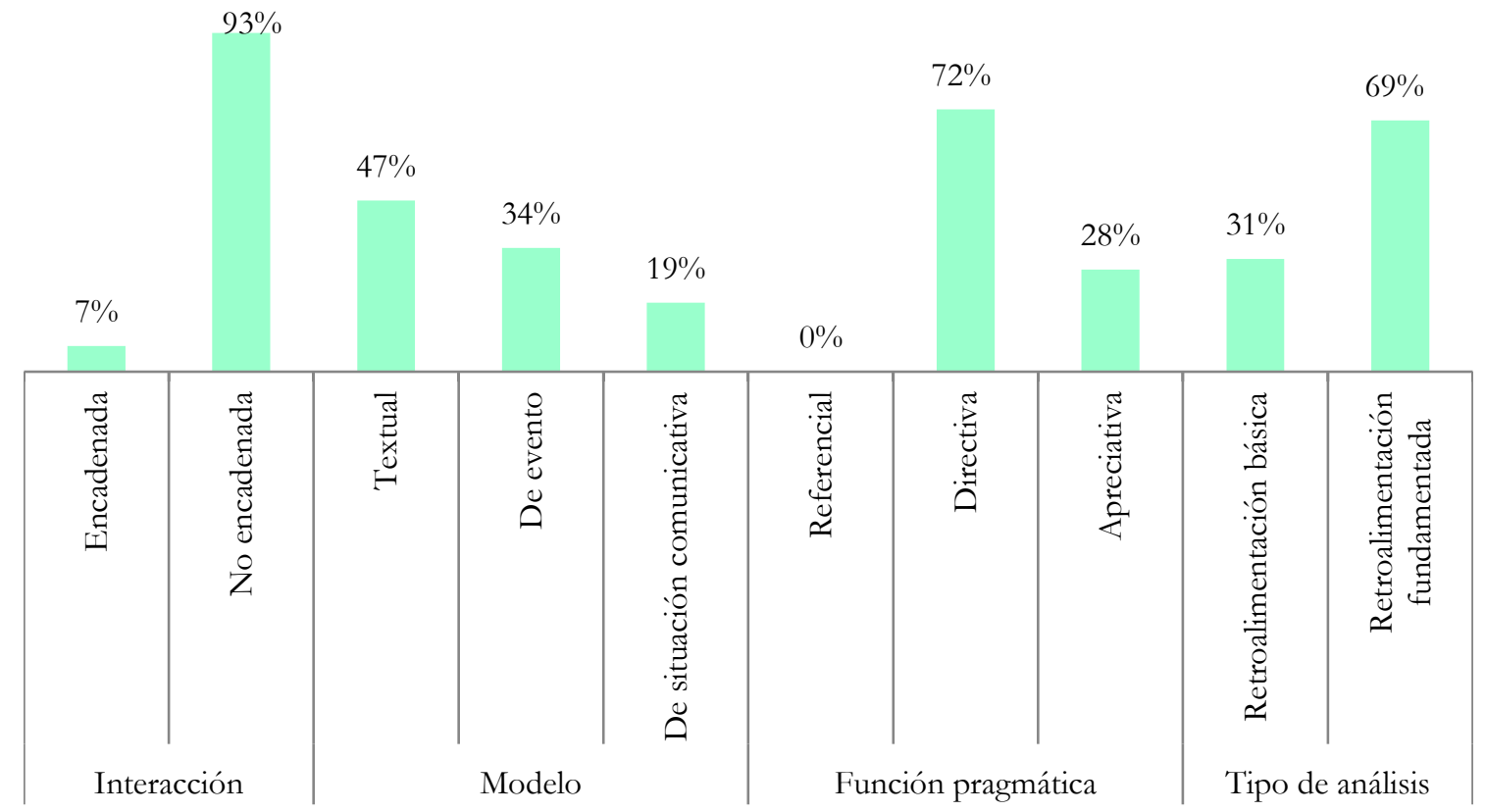

Fuente: elaboración propia.

Como se advierte, el encadenamiento ocupa un lugar mínimo en las retroalimentaciones globales (inexistente, como se ha señalado, en el caso de los comentarios en texto). En segundo lugar, es más homogénea la distribución de los porcentajes entre los tres tipos de modelo; al respecto aparecen sugerencias de interés sobre el modelo de situación comunicativa (e. g. «Puede ser conveniente reformular los títulos de los apartados, ya que es importante demostrar al lector especializado que uno se aleja de una lectura un tanto repetitiva del autor para abordar la comprensión hermenéutica y profundizar en su análisis») y sobre el modelo de evento («... quizá se repitan algunas ideas... me pareció leer varias veces lo mismo y sentí que el texto no avanzaba en la demostración de tu hipótesis»). No obstante, el modelo textual muestra preeminencia con significatividad estadística sobre el de situación comunicativa $(z=1,974 ; p=0,05)$. En tercer lugar, vuelve a prevalecer la función directiva, en este caso sobre la apreciativa $(\mathrm{z}=2,514 ; \mathrm{p}=0,05)$; ejemplificamos con un comentario que las incluye a ambas: «Sugiero la posibilidad de agregar un párrafo que anuncie los poemas que se tratarán a modo de operador/contextualizador previo. La exposición de las interpretaciones es clara y las secuencias conceptuales, bien marcadas». Por último, se advierte un mayor nivel de fundamentación $(z=2,067$; $\mathrm{p}=0,05)$; en este sentido, prácticamente se invierte la proporción de respuestas fundamentadas en comparación con los comentarios en texto.

\subsection{Asociación entre perfil del alumno y tipos de comentario}

A fin de analizar la asociación entre nuestra aproximación a los perfiles de los alumnos y su desempeño en la $\mathrm{R}$ en texto y global del capítulo del par interesa abordar el tipo de análisis (cfr. Tabla 6): 
Tabla 6.

Tabulación cruzada entre aproximación al perfil escritural y tipo de análisis

\begin{tabular}{llccc}
\hline \multirow{2}{*}{ Perfil } & & \multicolumn{2}{c}{ Retroalimentación } & Total \\
\hline Inferior-intermedio & Frecuencia & Básica & Fundamentada & \\
& Porcentaje & 28 & 3 & 31 \\
\multirow{2}{*}{ Intermedio } & Frecuencia & $6,5 \%$ & $1,9 \%$ & $5,3 \%$ \\
& Porcentaje & 314 & 94 & 408 \\
\multirow{2}{*}{ Intermedio-superior } & Frecuencia & $73,2 \%$ & $60,6 \%$ & $69,9 \%$ \\
& Porcentaje & 64 & 44 & 108 \\
\multirow{2}{*}{ Superior } & Frecuencia & $14,9 \%$ & $28,4 \%$ & $18,5 \%$ \\
& Porcentaje & 23 & 14 & 37 \\
& Total & $5,4 \%$ & $9 \%$ & $6,3 \%$ \\
& Frecuencia & 429 & 155 & 584 \\
& & $100 \%$ & $100 \%$ & $100 \%$ \\
\hline
\end{tabular}

Fuente: SPSS 22.0.

Estas diferencias resultan estadísticamente significativas $\left(X^{2}=20,679 ; g l=3 ; p=0,000\right)$. Como se observa, en el perfil inferior-intermedio priman los análisis básicos; en el intermedio, los porcentajes son similares; desde el intermedio-superior las retroalimentaciones fundamentadas prácticamente duplican a las básicas.

La asociación hallada se asocia con resultados previos (cfr. Álvarez y Difabio de Anglat, 2017b): los cursantes que confían en las propias habilidades escriturarias, valoran su importancia, monitorean el propio desempeño, conciben la producción del conocimiento de una manera interpretativa y/o constructiva y la escritura en términos de elaboración, son quienes proporcionan un mayor número de comentarios fundamentados. En cambio, categorías inferiores en dichas dimensiones se corresponden con una frecuencia mayor de R básica.

\section{Conclusiones}

Si bien se incrementa de modo progresivo la oferta de posgrados y la cantidad de estudiantes que los cursan, se registra un bajo porcentaje que finalmente se titula. Uno de los motivos de esta situación es la dificultad que encuentran los alumnos para elaborar la tesis, una tarea en general solitaria y con escasez de orientación didáctica.

En orden a contribuir a la solución de este problema, el presente artículo se orientó a analizar un área poco estudiada por la investigación previa: las intervenciones de estudiantes con diferentes perfiles escriturales en un seminario virtual sobre escritura respecto de capítulos avanzados de las tesis de posgrado de sus pares.

La aproximación a los perfiles deriva de una combinación ad hoc de nuestros instrumentos de diagnóstico, efectuada a través de análisis estadísticos y categorización de las respuestas a preguntas abiertas en el grupo total de participantes de nuestros talleres $(\mathrm{N}=74)$. La Clínica de escritura, por su parte, combina la plataforma Moodle con los capítulos compartidos en Google Drive a través de actividades andamiadas de manera externa (Carlino, 2008) y pautadas semanalmente. Gira en torno del análisis y la retroalimentación (R) en texto y global (Kumar y Stracke, 2007) del capítulo del par y del propio desde los modelos de situación comunicativa, de evento y textual (Cubo de Severino y Bosio, 2011). En este análisis, se ha aplicado un sistema categorial exhaustivo —encadenamiento o no, foco (los modelos referidos), función (comentario referencial, directivo o apreciativo, según Basturkmen et al., 2014) y tipo de análisis ( $\mathrm{R}$ básica o fundamentada) — a un corpus de 584 comentarios, a fin de establecer similitudes y diferencias entre las retroalimentaciones de los estudiantes de perfil similar y de perfil disímil.

Respecto de la $\mathrm{R}$ en texto, con significación estadística (prueba de diferencia de proporciones), la similitud reside en que prevalecen los comentarios referidos al modelo textual (que atiende, por otra parte, a los aspectos lingüísticos; es mínima la referencia a movimientos y pasos retóricos), con función directiva y desde una $\mathrm{R}$ básica. El primer resultado se equipara, entonces, con los hallazgos de 
Basturkmen et al. (2014), quienes informan que la exactitud lingǘstica es el aspecto más frecuente en los comentarios (del docente, en dicha investigación). La prevalencia de la función directiva podría explicarse por la actividad laboral de los cursantes: todos son profesores y, por ende, habituados a proporcionar recomendaciones a sus alumnos que pautan acciones futuras.

En las retroalimentaciones globales, si bien el porcentaje mayor sigue remitiendo al modelo textual, es más homogénea la distribución de los comentarios entre los tres tipos de modelo y, por otra parte, se advierte un nivel de fundamentación superior. En este sentido, de modo asimilable a los resultados de investigaciones previas, los foros de la Clínica de escritura parecen propiciar la conformación de una comunidad discursiva particular (Maher et al., 2008) en cuanto formalizada por ciertos usos del lenguaje en relación con la tesis de posgrado como género (incluso los alumnos que no provienen de las Letras van incorporado con naturalidad léxico especializado). Esta dinámica puede afianzar la revisión entre pares como proceso positivo y productivo al estimular el desarrollo de una mirada crítica (Carlino, 2008; DeLyser, 2003) que podría impactar en los escritos en cuanto los pares sugieren modificaciones necesarias (Colombo, 2013). Por otra parte, la mirada global del texto parecería suscitar más reflexiones en torno a las diferentes dimensiones de la tesis, sin hacer predominar los comentarios sobre los aspectos puramente lingüísticos.

Respecto de las diferencias en las retroalimentaciones de los cursantes, mediante la prueba de $\mathrm{Chi}^{2}$, en línea con estudios previos (Álvarez y Difabio de Anglat, 2017b), se halla la asociación esperada entre nuestra aproximación a los perfiles escriturales de los estudiantes y su desempeño en la $\mathrm{R}$ del capítulo del par: en el perfil inferior-intermedio priman los análisis básicos; en el intermedio, los porcentajes son similares para análisis básicos y fundamentados; desde el intermedio-superior las retroalimentaciones fundamentadas duplican a las básicas. Esta $\mathrm{R}$ fundamentada que, en lugar de correcciones directas, discute el contenido, incluye sugerencias de fondo, alternativa/s de análisis y/o preguntas orientadas a resolver problemas, puede favorecer la reflexión sobre la trama expositivo-argumentativa y, desde allí, la mejora sustancial del capítulo.

Dado que la novedad de esta Clínica es su carácter totalmente virtual, en la experiencia desarrollada se mostraría, como en Kozar y Lum (2015), el potencial de los grupos de escritura que interactúan a través de herramientas de comunicación mediadas por computadora. Al respecto, el documento compartido en Google Drive y los foros en Moodle favorecieron la actividad (e. g. aunque con gran dispersión, el número de intervenciones es apreciable) y una dinámica muy difícil de lograr en la presencialidad. En este sentido, una de las cursantes en la evaluación holística del curso afirmó: «Los aportes se vieron potenciados por el aprendizaje en línea, ya que cuando se trabaja con tanto detalle con textos complejos, lo más útil es el uso de herramientas de edición como Google Drive que van manteniendo en interacción y actualizados a los participantes. Creo que en un entorno presencial, tal grado de minuciosidad en el trabajo no hubiera resultado nunca tan logrado».

A nuestro juicio, los resultados obtenidos muestran un camino beneficioso para la enseñanza y el aprendizaje de la escritura de la tesis mediados por tecnologías digitales: al propiciar la tarea escritural como cuestión pública y trabajo compartido (Carlino, 2005; DeLyser, 2003; Maher et al., 2008) en un espacio virtual ágil y operativo (Kozar y Lum, 2015), el grupo de escritura favorece la deliberación sostenida sobre las diferentes dimensiones del género y sobre las estrategias para su elaboración, al tiempo que se promueve una reflexión metalingüística que puede «motorizar» la revisión-edición del texto y la elaboración futura.

No obstante, es necesario profundizar el análisis porque, si bien por un lado hemos identificado aspectos relevantes en la dinámica de la $\mathrm{R}$ del par $\mathrm{y}$, por otro, la asociación entre tipo de comentario y perfil aproximado del alumno, resta analizar: 1) la reflexión de cada estudiante sobre su propio capítulo; 2) la incidencia de la $\mathrm{R}$ y sus tipos en la mejora del texto, en las modificaciones efectivamente realizadas por cada cursante en la versión definitiva del capítulo que opera como evaluación final del curso; y 3) las relaciones entre perfil aproximado del alumno y ocurrencia de dichas mejoras, estudio que puede habilitar un seguimiento todavía más cercano de los estudiantes con bajas puntuaciones en el diagnóstico. Además, es forzoso ampliar la muestra a fin de ponderar la consistencia del procedimiento de adscripción de los perfiles y de estos primeros hallazgos.

En continuidad con esta línea investigativa interesa, por un lado, analizar la $\mathrm{R}$ que aporta el director y/o el codirector de la tesis al documento con todos los comentarios del par y de las tutoras del taller y su incidencia en la versión final. Por otro, elaborar y aplicar grillas de evaluación, al mismo tiempo integrales y estratégicas, para cada capítulo de la tesis (los cursantes suelen someter a $\mathrm{R}$ sobre todo capítulos teóricos, pero también metodológicos y de resultados). Desde la aplicación de estas grillas 
se podrán meta-evaluar las consignas que guían la $\mathrm{R}$ en texto y global de cada una de las tres fases de la Clínica a fin de fortalecer la mejor promoción posible de la reflexión (epistemológica y metalingüística) y de la revisión del escrito.

\section{Referencias}

Aitchison, C., \& Lee, A. (2006). Research writing: Problems and pedagogies. Teaching in Higher Education, 11(3), pp. 265-278. doi: 10.1080/13562510600680574

Álvarez, G., \& Difabio de Anglat, H. (2016). Formación virtual en estrategias para la producción conceptual y escrita en el posgrado en Ciencias Sociales y Humanas. Revista Q, 10(20), pp. 110-136. doi: $10.18566 /$ revistaq.v10n20.a05

Álvarez, G., \& Difabio de Anglat, H. (2017a). La actividad metalingüística en espacios de interacción entre pares: reflexiones en torno a un taller virtual orientado a la escritura de la tesis de posgrado. Perfiles educativos, XXXIX(155), pp. 51-67. Recuperado de https://www.redalyc.org/pdf/132/13250922004.pdf.

Álvarez, G., \& Difabio de Anglat, H. (2017b). Perfil del estudiante y nivel de construcción del conocimiento en intervenciones en foros. Reflexiones en torno a un taller virtual de tesis en el posgrado. En A. H. González, \& M. Martin (Comps.), $4^{\circ}$ Jornadas de TIC e Innovación en el Aula: Más Allá del Aula Virtual. Otros Horizontes, otros desafios (pp. 600-610). La Plata: Dirección General de Educación a Distancia y Tecnologías, Universidad Nacional de La Plata. Recuperado de http://www.fi.unsj.edu.ar/descargas/noticias/Libro\%20Cuartas\%20Jornadas\%20TICS.pdf.

Álvarez, G., \& Difabio de Anglat, H. (2018). Retroalimentación docente y aprendizaje en talleres virtuales de escritura de tesis. Apertura. Revista de innovación educativa, 10(1), pp. 8-23. doi: 10.32870/Ap.v10n1.996

Arnoux, E., Borsinger de Montemayor, A., Carlino, P., Di Stefano, M., Pereira, C., \& Silvestre, A. (2004). La intervención pedagógica en el proceso de escritura de tesis de postgrado. Revista de la Maestría en Salud Pública, 2(3), pp. 1-16. Recuperado de https://www.aacademica.org/paula.carlino/169.pdf.

Basturkmen, H., East, M., \& Bitchener, J. (2014). Supervisors' on-script feedback comments on drafts of dissertations: socialising students into the academic discourse community. Teacbing in Higher Education, 19(4), pp. 432-445. doi: 10.1080/13562517.2012.752728

Bitchener, J., Basturkmen, H., \& East, M. (2010). The Focus of Supervisor Written Feedback to Thesis/Dissertation Students. International Journal of English Studies, 10(2), pp. 79-99. doi: 10.6018/ijes/2010/2/119201

Boud, D., \& Lee, A. (2005). Peer learning as pedagogic discourse for research education. Studies in Higher Education, 30(5), pp. 501-515. doi: 10.1080/03075070500249138

Bourdieu, P. (1976). El campo científico. Buenos Aires: Nueva Visión.

Caffarella, R., \& Barnett, B. (2000). Teaching Doctoral Students to Become Scholarly Writers: The importance of giving and receiving critiques. Studies in Higher Education, 25(1), pp. 39-52. doi: 10.1080/030750700116000

Carlino, P. (2005). ¿Por qué no se completan las tesis en los posgrados? Obstáculos percibidos por maestrandos en curso y magister exitosos. Educere, Revista Venezolana de Educación, 9(30), pp. 415420. Recuperado de https://www.aacademica.org/paula.carlino/15.pdf.

Carlino, P. (2008). Exploración de géneros, diarios de tesis y revisión entre pares. En E. Arnoux (Dir.), Escritura y producción de conocimientos en las carreras de posgrado (pp. 227-246). Buenos Aires: Santiago Arcos Editor.

Castelló, M., González, D., \& Iñesta, A. (2010). La regulación de la escritura académica en el doctorado: el impacto de la revisión colaborativa en los textos. Revista Española de Pedagogía, 68(247), pp. 521-537. Recuperado de https://revistadepedagogia.org/wp-content/uploads/2010/11/24706.pdf.

Colombo, L. (2013). Una experiencia pedagógica con grupos de escritura en el posgrado. Aula Universitaria, 15, pp. 61-68. doi: 10.14409/au.v1i15.4368

Colombo, L., \& Carlino, P. (2015). Grupos para el desarrollo de la escritura científico-académica: Una revisión de trabajos anglosajones. Lenguaje, 43(1), pp. 13-34. 10.25100/lenguaje.v43i1.4993 
Cubo de Severino, L., \& Bosio, I. V. (2011). La tesis como clase textual y su proceso de escritura. En L. Cubo, H. Puiatti, \& N. Lacon (Eds.), Escribir una tesis. Manual de estrategias de producción (pp. 1134). Córdoba: Comunic-arte.

Delamont, S. (2005). Four great gates: dilemmas, directions and distractions in educational research. Research Papers in Education, 20(1), pp. 85-100. doi: 10.1080/0267152052000341345

DeLyser, D. (2003). Teaching graduate students to write: a seminar for thesis and dissertation writers. Journal of Geography in Higher Education, 27(2), pp. 169-181. doi: 10.1080/03098260305676.

Difabio de Anglat, H. (2012). Hacia un inventario de escritura académica en el posgrado. Revista de Orientación Educacional, 26(49), pp. 37-53. Recuperado de https://dialnet.unirioja.es/descarga/articulo/4554495.pdf.

Difabio de Anglat, H., \& Álvarez, G. (2017). Alfabetización académica en entornos virtuales: Estrategias para la promoción de la escritura de la tesis de posgrado. Traslaciones. Revista Latinoamericana de Lectura y Escritura, 4(8), pp. 97-120. Recuperado de:

http://revistas.uncu.edu.ar/ojs/index.php/traslaciones/issue/view/83.

Difabio de Anglat, H., \& Heredia, M. del V. (2013). El taller de tesis doctoral en educación desde un enfoque comprehensivo de escritura a través de la plataforma Moodle. En: $6^{\circ}$ Seminario Internacional de Educación a Distancia. Recuperado de http://www.uncu.edu.ar/seminario_rueda/upload/t234.pdf.

Difabio de Anglat, H., Portela de Nieto, A., Gelonch Villarino, S., Muscará, F., \& Boarini, M. G. (2018). La experiencia de investigación educativa de nivel doctoral en la región de Cuyo, Argentina. REDU. Revista de Docencia Universitaria, 16(1), pp. 11-32. doi: 10.4995/redu.2018.5690

Hernández Sampieri, R., Fernández Collado, C., \& Baptista Lucio, P. (2014). Metodología de la investigación ( $6^{\mathrm{a}}$ ed.). México: McGraw Hill.

Hofer, B. (2004). Epistemological Understanding as a Metacognitive Process: Thinking Aloud During Online Searching. Educational Psychologist, 39(1), pp. 43-55. doi: 10.1207/s15326985ep3901_5

Jara Solar, I. (2013). Descripción funcional de introducciones de tesis doctorales en las disciplinas de química y lingüística. Onomázein. Revista semestral de lingïística, filología y traducción, 28, pp. 72-87. doi: 10.7764/onomazein. 28.7

Kozar, O., \& Lum, J. F. (2015). Online doctoral writing groups: do facilitators or communication modes make a difference? Quality in Higher Education, 21(1), pp. 38-51. doi: $10.1080 / 13538322.2015 .1032003$

Kumar, V., \& Stracke, E. (2007). An analysis of written feedback on a PhD thesis. Teaching in Higher Education, 12(4), pp. 461-470. doi: 10.1080/13562510701415433

Lassig, C., Lincoln, M., Dillon, L., Diezmann, C., Fox, J., \& Neofa, Z. (2009). Writing together, learning together: the value and effectiveness of a research writing group for doctoral students. En: Australian Association for Research in Education 2009 International Education Research Conference, 29 November - 3 December, 2009, National Convention Centre, Canberra. Recuperado de https://eprints.qut.edu.au/28976/1/c28976.pdf.

Li, S., \& Seale, C. (2007). Managing Criticism in PhD Supervision: A Qualitative Case Study. Studies in Higher Education, 32(4), pp. 511-526. doi: 10.1080/03075070701476225

Maher, D., Seaton, L., McMullen, C., Fitzgerald, T., Otsuji, E., \& Lee, A. (2008). Becoming and being writers: the experiences of doctoral students in writing groups. Studies in Continuing Education, 30(3), pp. 263-275. doi: 10.1080/01580370802439870

Martínez-Fernández, J. R. (2004). Concepción de aprendizaje, metacognición y cambio conceptual en estudiantes universitarios de Psicología (Tesis de Doctorado). Universidad Autónoma de Barcelona, España. Recuperado de http://www.tdx.cat/handle/10803/2632.

Martínez-Fernández, J. R. (2007). Concepción de aprendizaje y estrategias metacognitivas en estudiantes universitarios de psicología. Anales de Psicología, 23(1), pp. 7-16. Recuperado de: http://www.redalyc.org/articulo.oa?id=16723102.

Pozo, J. I., \& Scheuer, N. (1999). Las concepciones sobre el aprendizaje como teorías implícitas. En J. I. Pozo, \& C. Monereo (Coords.), El aprendizaje estratégico (pp. 87-108). Madrid: Santillana.

Rebollo Quintela, N., \& Espiñeira Bellón, E. M. (2017). La tutoría durante el proceso de desarrollo del TFG y TFM: análisis del grado de utilidad y satisfacción del alumnado. Educatio Siglo XXI, 35(2), pp. 161-180. doi: 10.6018/j/298561 
San Mateo-Valdehíta, A., Escobar-Álvarez, M. A., \& Chacón-Beltrán, R. (2018). El Trabajo de Fin de Máster (TFM) de Humanidades en el EEES: Análisis de las necesidades de los estudiantes. REDU. Revista de Docencia Universitaria, 16(1), pp. 303-319. doi: 10.4995/redu.2018.9873

Scardamalia, M., \& Bereiter, C. (1992). Dos modelos explicativos de los procesos de producción escrita. Infancia y Aprendizaje, 58, pp. 43-64. doi: 10.1080/02103702.1992.10822332

Stake, R. E. (2005). Qualitative case study. In N. K. Denzin, \& Y. S. Lincoln (Eds.), The Sage Handbook of Qualitative Research ( $2^{\mathrm{a}}$ ed.) (pp. 443-458). Thousand Oaks, CA: Sage.

Stake, R. E. (2010). Qualitative Research. New York: The Guildford Press.

Starke-Meyerring, D., Paré, A., Sun, K. Y., \& El-Bezre, N. (2014). Probing normalized institutional discourses about writing: The case of the doctoral thesis. Journal of Academic Language and Learning, 8(2), pp. 13-27. Recuperado de journal.aall.org.au/index.php/jall/article/download/295/195.

Vermunt, J. (1994). Inventory of Learning Styles in bigher education: Scoring key. Tilburg University: Department of Educational Psychology. doi: 10.1037/t14424-000 